Proyecciones Journal of Mathematics

Vol. 37, $\mathrm{N}^{o}$ 1, pp. 45-55, March 2018.

Universidad Católica del Norte

Antofagasta - Chile

\title{
A variant of the quadratic functional equation on semigroups
}

\author{
B. Fadli \\ IBN Tofail University, Morocco \\ D. Zeglami \\ Moulay ISMAIL University, Morocco \\ and \\ S. Kabbaj \\ IBN Tofail University, Morocco \\ Received: January 2017. Accepted : April 2017
}

\begin{abstract}
Let $S$ be a semigroup, let $H$ be an abelian group which is uniquely 2-divisible, and let $\sigma$ be an involutive automorphism of $S$. We express the solutions $f: S \rightarrow H$ of the following variant of the quadratic functional equation

$$
f(x y)+f(\sigma(y) x)=2 f(x)+2 f(y), \quad x, y \in S,
$$

in terms of bi-additive maps and solutions of the symmetrized additive Cauchy equation.
\end{abstract}

Subjclass [2010] : Primary 39B52.

Keywords : Symmetrized additive Cauchy equation, quadratic equation, additive function, semigroup. 


\section{Set up, notation and terminology}

Throughout the paper we work in the following framework and with the following notation and terminology. We use it without explicit mentioning.

$S$ is a semigroup [a set equipped with an associative composition rule $(x, y) \mapsto x y], \sigma: S \rightarrow S$ is an homomorphism satisfying $\sigma \circ \sigma=i d$, and $(H,+)$ denotes an abelian group which is uniquely 2-divisible, i.e., for any $h \in H$ the equation $2 x=h$ has exactly one solution $x \in H$.

A function $a: S \rightarrow H$ is said to be additive if

$$
a(x y)=a(x)+a(y) \text { for all } x, y \in S .
$$

A function $f: S \rightarrow H$ is abelian, if

$$
f\left(x_{\pi(1)} x_{\pi(2)} \cdots x_{\pi(k)}\right)=f\left(x_{1} x_{2} \cdots x_{k}\right)
$$

for all $x_{1}, x_{2}, \ldots, x_{k} \in S$, all permutations $\pi$ of $k$ elements and all $k=$ $2,3, \ldots$ Any abelian function $f$ is central, meaning $f(x y)=f(y x)$ for all $x, y \in S$.

By $\mathcal{N}(S, H, \sigma)$ we mean the set of the solutions $\theta: S \rightarrow H$ of the homogeneous equation

$$
\theta(x y)-\theta(\sigma(x) y)=0, \quad x, y \in S .
$$

We recall that the Cauchy difference $C f$ of a function $f: S \rightarrow H$ is defined by

$$
C f(x, y):=f(x y)-f(x)-f(y), \quad x, y \in S .
$$

If $f: S \rightarrow H$ is a function, then $J_{f}: S \rightarrow H$ and $\varphi_{f}: S \rightarrow H$ are defined by

$$
2 f(x):=J_{f}(x)+C f(x, x) \text { and } \quad \varphi_{f}(x):=f(\sigma(x) x)
$$

for all $x \in S$.

\section{Introduction}

The purpose of the present paper is to solve the following variant of the quadratic functional equation

$$
f(x y)+f(\sigma(y) x)=2 f(x)+2 f(y), \quad x, y \in S,
$$


where $f: S \rightarrow H$ is the unknown function. The difference between (2.1) and the quadratic standard functional equation

$$
f(x y)+f(x \sigma(y))=2 f(x)+2 f(y), \quad x, y \in S,
$$

is that the new equation (2.1) has, on the second term, $f(\sigma(y) x)$ while the old one $(2.2)$ has $f(x \sigma(y))$. On abelian semigroups the functional Eqs. (2.1) and (2.2) coincide, and their solutions are known see e.g. [10] and [13, Chapter 13], so the contributions of the present paper to the theory of quadratic functional equations lie in the non-abelian case.

A special case of (2.1) is the symmetrized additive Cauchy equation

$$
f(x y)+f(y x)=2 f(x)+2 f(y), \quad x, y \in S .
$$

Eq. (2.3) is a non-commutative version of the additive equation (1.1), because it reduces to (1.1) if $S$ is abelian. On groups the solutions of (2.3) are according to [13, Proposition 2.17] the same as those solutions $f$ of Jensen's functional equation $f(x y)+f\left(x y^{-1}\right)=2 f(x)$ for which $f(e)=$ 0. Example 12.4 in [13] present a non-abelian solution of (2.3) on the 3dimensional Heisenberg group $H_{3}(\mathbf{R})$. Therefore the functional equation (2.1) has in general non-abelian solutions.

Similar functional equations that have also been studied are

$$
\begin{aligned}
& f(x y)+f(\sigma(y) x)=2 f(x) f(y), \quad x, y \in S, \\
& f(x y)+f(\sigma(y) x)=2 f(x) g(y), \quad x, y \in S, \\
& f(x y)+f(\sigma(y) x)=2 f(x), \quad x, y \in S .
\end{aligned}
$$

The complex-valued solutions of (2.4) were determined by Stetkær in [14], while the complex-valued solutions $(f, g)$, where $S$ is a possibly nonabelian group or monoid, of (2.5) and the solutions $f: S \rightarrow H$ of (2.6) were obtained by the authors in [3] and [4], respectively. It turns out that, like on abelian groups, only multiplicative and additive functions occur in the solution formulas of (2.4), (2.5) and (2.6). We will prove that these contrast the solutions of the functional equation (2.1), where the non-abelian phenomena like solutions of (2.3) may occur. For other similar functional equations we refer to $[1,2,5,7-9,11,12,16]$.

One of the main results is that the solutions for the variant (2.1) of the quadratic functional equation can be expressed in terms of bi-additive maps and solutions of the symmetrized additive Cauchy equation (Theorem 5.4), so that the form of the solutions generalizes the case where $S$ is abelian, see e.g. $[10$, Theorem 3] and [13, Theorem 13.6]. Thus the contribution by our 
paper of new knowledge is an extension of earlier results from the abelian to the non-abelian case because (2.3) becomes (1.1) if $S$ is abelian.

As applications, two important results (Corollaries 5.3 and 5.6 about Drygas' type equation

$$
f(x y)+f(\sigma(y) x)=2 f(x)+f(y)+f(\sigma(y)), \quad x, y \in S,
$$

are presented. Our solution formulas contain the abelian ones as special cases.

Finally, we note that the results about Whitehead's functional equation

$$
f(x y z)=f(x y)+f(x z)+f(y z)-f(x)-f(y)-f(z), \quad x, y, z \in S,
$$

given in [15] play an important role in finding solutions to the functional equation (2.1).

\section{Results about Whitehead's functional equation (2.7)}

The following lemma lists pertinent basic properties of any solution $f$ : $S \rightarrow H$ of $(2.7)$. For the notation $J_{f}$, see Section 1 .

Theorem 3.1. Let $f: S \rightarrow H$ be a solution of (2.7). In that case

(a) $C f: S \times S \rightarrow H$ is bi-additive.

(b) $J_{f}: S \rightarrow H$ satisfies (2.3).

(c) If $f$ is central, then $J_{f}$ is additive.

(d) Let $s \in \operatorname{Hom}(S, S)$. If $f \circ s=f$, then $J_{f} \circ s=J_{f}$.

Proof. $\quad(a)$ and $(b)$ can be found in [15]

(c) Let $f$ be central. To get that $J_{f}$ is central it suffices to prove that so is $x \mapsto C f(x, x)$. That is an easy task. The rest follows from $(b)$.

(d) By the definition of $J_{f}$ it suffices to prove that $C f(s(x), s(x))=$ $C f(x, x)$ for all $x \in S$, and that follows from the definition of $C f$. 


\section{Connections between (2.1) and (2.7)}

Lemma 4.1 below derives one connection between (2.1) and (2.7), viz., Lemma 4.1. If $f: S \rightarrow H$ satisfies (2.1), then it also satisfies (2.7).

Proof. Making the substitutions $(x y, z),(\sigma(z) x, y)$, and $(x, y z)$ in $(2.1)$, we get respectively

$$
\begin{aligned}
f(x y z)+f(\sigma(z) x y) & =2 f(x y)+2 f(z), \\
f(\sigma(z) x y))+f(\sigma(y z) x) & =2 f(\sigma(z) x)+2 f(y), \\
f(x y z)+f(\sigma(y z) x) & =2 f(x)+2 f(y z) .
\end{aligned}
$$

Subtracting the middle identity from the sum of the other two we find that

$2 f(x y z)=2 f(x y)+2 f(y z)+2 f(x)+2 f(z)-2 f(\sigma(z) x)-2 f(y)$.

Replacing here $f(\sigma(z) x)$ by $2 f(x)+2 f(z)-f(x z)$ and using the fact that $H$ is uniquely 2-divisible, we get (2.7).

In the following lemma, we derive another connection between (2.1) and $(2.7)$.

Lemma 4.1. If $f: S \rightarrow H$ satisfies (2.1), then $\varphi_{f}=J_{f}$.

Proof. The proof is a small computation, based on (2.1).

$$
\begin{aligned}
\varphi_{f}(x)-J_{f}(x) & =f(\sigma(x) x)-[2 f(x)-C f(x, x)] \\
& =f(\sigma(x) x)+f(x x)-f\left(x^{2}\right)-\left[2 f(x)-f\left(x^{2}\right)+2 f(x)\right] \\
& =2 f(x)+2 f(x)-4 f(x)=0 .
\end{aligned}
$$

\section{Results about (2.1)}

We start with Lemma 5.1, in which we derive some properties of the solutions of (2.1).

Lemma 5.1. If $f: S \rightarrow H$ satisfies (2.1), then 
a) $f \circ \sigma=f$.

b) $C f(x, \sigma(y))=-C f(y, x)$ for all $x, y \in S$.

c) $\varphi_{f}$ is a solution of (2.1).

d) $\varphi_{f} \in \mathcal{N}(S, H, \sigma)$, and $\varphi_{f} \circ \sigma=\varphi_{f}$.

Proof. (a) Let $x, y \in S$ be arbitrary. Using (2.7) and (2.1), we obtain

$$
\begin{aligned}
f(\sigma(y) x y) & =f(\sigma(y) x)+f(\sigma(y) y)+f(x y)-f(\sigma(y))-f(x)-f(y) \\
& =f(\sigma(y) y)+[f(x y)+f(\sigma(y) x)]-f(\sigma(y))-f(x)-f(y) \\
& =f(\sigma(y) y)+f(x)+f(y)-f(\sigma(y)) .
\end{aligned}
$$

On the other hand, we have

$$
\begin{aligned}
f(\sigma(y) x y) & =2 f(x y)+2 f(y)-f\left(x y^{2}\right) \\
& =2 f(x y)+2 f(y)-\left[2 f(x y)+f\left(y^{2}\right)-f(x)-2 f(y)\right] \\
& =4 f(y)-f\left(y^{2}\right)+f(x) \\
& =f(\sigma(y) y)+f(x) .
\end{aligned}
$$

So $f(y)-f(\sigma(y))=0$ for all $y \in S$, i.e., $f \circ \sigma=f$.

(b) Let $x, y \in S$ be arbitrary. By help of (a) and (2.1), we get that

$$
\begin{aligned}
C f(x, \sigma(y)) & =f(x \sigma(y))-f(x)-f(\sigma(y)) \\
& =f(\sigma(x) y)-f(x)-f(y) \\
& =2 f(y)+2 f(x)-f(y x)-f(x)-f(y) \\
& =f(y)+f(x)-f(y x) \\
& =-C f(y, x) .
\end{aligned}
$$

In $(c)$ and $(d)$ we use a couple of times that $\varphi_{f}=J_{f}$ (Lemma 4.2).

(c) Recalling the definition of $J_{f}$ (Section 1) we get that $2 f(x)=$ $C f(x, x)+\varphi_{f}(x)$, so by linearity it suffices to show that the function $x \mapsto C f(x, x)$ is a solution of (2.1). And that is a simple computation, based on the bi-additivity of $C f$ and $(b)$. 
(d) We read from Theorem 3.1(b) that $\varphi_{f}(x y)+\varphi_{f}(y x)=2 \varphi_{f}(x)+$ $2 \varphi_{f}(y)$ for all $x, y \in S$. Comparing this with $(c)$ gives the first statement of $(d)$. That $\varphi_{f} \circ \sigma=\varphi_{f}$ is a special instance of Theorem 3.1(d).

In the following theorem, we determine the central solutions $f: S \rightarrow H$ of the functional equation (2.1). For abelian case it generalizes many results (see, e.g., [10,Theorem 3] and [13, Theorem 13.6]).

Theorem 5.2. The central solutions $f: S \rightarrow H$ of (2.1) are the functions of the form

$$
f(x)=Q(x, x)+a(x)
$$

where $Q: S \times S \rightarrow H$ is an arbitrary symmetric, bi-additive map such that $Q(x, \sigma(y))=-Q(x, y)$ for all $x, y \in S$, and where $a: S \rightarrow H$ is an arbitrary additive map such that $a \circ \sigma=a$.

Proof. Assume that $f: S \rightarrow H$ is a central solution of (2.1). Since $f$ is central, then $C f$ is symmetric and $\varphi_{f}$ is additive (Theorem $3.1(c)$ ). So $f$ has the desired form by the decomposition $2 f(x)=C f(x, x)+\varphi_{f}(x)$. Take $Q=\frac{1}{2} C f$ and $a=\frac{1}{2} \varphi_{f}$.

The other direction of the proof is trivial to verify.

As a consequence of Theorem 5.2, we have the following result on the central solutions of the functional equation

$$
f(x y)+f(\sigma(y) x)=2 f(x)+f(y)+f(\sigma(y)), \quad x, y \in S,
$$

which reveals a connection between (5.1) and (2.1) and contains the solution of Drygas' equation on commutative semigroups.

Corollary 5.3. The central solutions $f: S \rightarrow H$ of (5.1) are the functions of the form

$$
f(x)=Q(x, x)+a(x)
$$

where $Q: S \times S \rightarrow H$ is an arbitrary symmetric, bi-additive map such that $Q(x, \sigma(y))=-Q(x, y)$ for all $x, y \in S$, and where $a: S \rightarrow H$ is an arbitrary additive map. 
Proof. It is easy to check that any function $f$ of the form (5.2) is central and satisfies (5.1). Conversely, assume that $f$ is a central solution of (5.1). Let $f_{e}$ and $f_{o}$ denote the $\sigma$-even and the $\sigma$-odd parts of $f$, i.e.,

$$
f_{e}=\frac{f+f \circ \sigma}{2} \text { and } f_{o}=\frac{f-f \circ \sigma}{2} .
$$

Simple computations show that $f_{e}$ is a central solution of (5.1). Hence $f_{e}$ is a central solution of (2.1). From Theorem 5.2, we see that there exist a symmetric, bi-additive map $Q: S \times S \rightarrow H$ with $Q(x, \sigma(y))=-Q(x, y)$ for all $x, y \in S$, and an additive map $a_{1}: S \rightarrow H$ with $a_{1} \circ \sigma=a_{1}$ such that

$$
f_{e}(x)=Q(x, x)+a_{1}(x), \quad x \in S .
$$

On the other hand, since $f_{o}=f-f_{e}, f_{o}$ is also a solution of (5.1), so that $f_{o}$ is a solution of the variant (2.6) of Jensen's functional equation. According to $\left[4\right.$, Theorem 3.2], we see that $f_{o}$ is additive. Therefore $f=$ $f_{e}+f_{o}$ has the required form with $a=a_{1}+f_{o}$ and this completes the proof.

Now we treat the general case where the solution $f: S \rightarrow H$ need not be central.

Theorem 5.4. The general solution $f: S \rightarrow H$ of $(2.1)$ is

$$
f(x)=Q(x, x)+\psi(x), \quad x \in S,
$$

where $Q: S \times S \rightarrow H$ is an arbitrary bi-additive map such that $Q(x, \sigma(y))=$ $-Q(y, x)$ for all $x, y \in S$, and where $\psi: S \rightarrow H$ is an arbitrary solution of the symmetrized additive Cauchy equation such that $\psi \circ \sigma=\psi$ and $\psi \in \mathcal{N}(S, H, \sigma)$.

Proof. Let $f: S \rightarrow H$ be a solution of (2.1). Using the decomposition $2 f(x)=C f(x, x)+\varphi_{f}(x)$, Theorem 3.1 and Lemma 5.1, we see that $f$ has the desired form. Take $Q=\frac{1}{2} C f$ and $\psi=\frac{1}{2} \varphi_{f}$.

The other direction of the proof is trivial to verify.

Remark 5.1. Theorem 5.4 is a non-abelian version of e.g. [10,Theorem 3] and [13, Theorem 13.6]. Indeed, if $S$ is abelian, then any solution of the symmetrized additive Cauchy equation reduces to an additive function and so the condition $\psi \in \mathcal{N}(S, H, \sigma)$ becomes $\psi=\psi \circ \sigma$. 
In view of Theorem 5.4, we obtain the following result about solution, that need not be central, of Drygas' type equation (5.1) on any semigroup.

Corollary 5.2. The general solution $f: S \rightarrow H$ of (5.1) is

$$
f(x)=Q(x, x)+\psi(x)+a(x), \quad x \in S,
$$

where $Q: S \times S \rightarrow H$ is an arbitrary bi-additive map such that $Q(x, \sigma(y))=$ $-Q(y, x)$ for all $x, y \in S, \psi: S \rightarrow H$ is an arbitrary solution of the symmetrized additive Cauchy equation such that $\psi \circ \sigma=\psi$ and $\psi \in \mathcal{N}(S, H, \sigma)$, and where $a: S \rightarrow H$ is an additive function such that $a \circ \sigma=-a$.

Proof. As the proof of Corollary 5.3 with the necessary changes.

Acknowledgement. We wish to express our thanks to the referees for useful comments.

\section{References}

[1] H. Drygas, Quasi-inner products and their applications, in: Advances in Multivariate Statistical Analysis (ed. A. K. Gupta), D. Reidel Publishing Co., pp. 13-30, (1987).

[2] B. Fadli, D. Zeglami and S. Kabbaj, On a Gajda's type quadratic equation on a locally compact abelian group, Indagationes Math. 26 (4), pp. 660-668, (2015).

[3] B. Fadli, D. Zeglami and S. Kabbaj, A variant of Wilson's functional equation, Publ. Math. Debrecen 87 (3-4), pp. 415-427, (2015).

[4] B. Fadli, D. Zeglami and S. Kabbaj, A variant of Jensen's functional equation on semigroups, Demonstratio Math. 49 (4), pp. 413-420, (2016).

[5] B. Fadli, A. Chahbi, Iz. EL-Fassi and S. Kabbaj, On Jensen's and the quadratic functional equations with involutions, Proyecciones (Antofagasta) 35 (2), pp. 213-223, (2016). 
[6] V. A. Fă̌ziev and P. K. Sahoo, Solution of Whitehead equation on groups, Math. Bohem. 138(2), pp. 171-180, (2013).

[7] PL. Kannappan, Functional equations and inequalities with applications, Springer, New York, (2009).

[8] C.T. Ng, Jensen's functional equation on groups, III, Aequationes Math. 62 (1-2), pp. 143-159, (2001).

[9] P. de Place Friis and H. Stetkær, On the quadratic functional equation on groups, Publ. Math. Debrecen 69 (1-2), pp. 65-93, (2006).

[10] P. Sinopoulos, Functional equations on semigroups, Aequationes Math. 59(3), pp. 255-261, (2000).

[11] H. Stetkær, Functional equations on abelian groups with involution, Aequationes Math. 54 (1-2), pp. 144-172, (1997).

[12] H. Stetkær, On a variant of Wilson's functional equation on groups, Aequationes Math. 68 (3), pp. 160-176, (2004).

[13] H. Stetkær, Functional Equations on Groups, World Scientific Publishing Co. Pte. Ltd., Hackensack, NJ, (2013).

[14] H. Stetkær, A variant of d'Alembert's functional equation, Aequationes Math. 89 (3), pp. 657-662, (2015).

[15] H. Stetkær, The kernel of the second order Cauchy difference on semigroups, Aequationes Math. (2016). DOI 10.1007/s00010-016-0453-8.

[16] D. Zeglami, B. Fadli and S. Kabbaj, On a variant of $\mu$-Wilson's functional equation on a locally compact group, Aequationes Math. 89 (5), pp. 1265-1280, (2015). 


\section{B. Fadli}

Department of Mathematics, Faculty of Sciences, IBN TOFAIL University, B. P. : 14000. Kenitra, Morocco

e-mail : himfadli@gmail.com

\section{Zeglami}

Department of Mathematics, E. N. S. A. M, Moulay ISMAIL University, B. P: 15290 Al Mansour, Meknes, Morocco

e-mail : zeglamidriss@yahoo.fr and

\section{S. Kabbaj}

Department of Mathematics, Faculty of Sciences, IBN TOFAIL University, B. P. : 14000. Kenitra, Morocco

e-mail : samkabbaj@yahoo.fr 